INPLASY

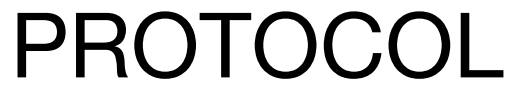

To cite: Wang et al. Can speed bump sign be a diagnostic tool for acute appendicitis?

Evidence-based appraisal by meta-analysis. Inplasy protocol 2021110052. doi:

10.37766/inplasy2021.11.0052

Received: 15 November 2021

Published: 15 November 2021

Corresponding author:

Yen-Ta Huang

uncleda.huang@gmail.com

Author Affiliation:

National Cheng Kung

University Hospital, College of

Medicine, National Cheng

Kung University, Tainan,

Taiwan.

Support: No financial support.

Review Stage at time of this submission: Data analysis Completed but not published.

\section{Can speed bump sign be a diagnostic tool for acute appendicitis? Evidence- based appraisal by meta-analysis}

Review question / Objective: Patient: patients with abdominal pain in emergency department; Intervention: speed bump sign;Comparison: acute appendicitis diagnosed by histology; $O$ : sensitivity, specificity, diagnostic odds ratio, likelihood ratio.

Condition being studied: Speed bumps are frequently used traffic device to slow down speed of vehicles and the "speed bump sign" is a clinical symptom with aggravated abdominal pain while driving over speed bumps, whose reason bases on irritation of inflamed peritoneum while moving. It is used by some doctors taking history from abdominal pain cases and the aggravation of pain while travelling over speed bumps is thought to be associated with an increased likelihood of acute appendicitis. However, the certainty of its evidence has not been validated.

INPLASY registration number: This protocol was registered with the International Platform of Registered Systematic Review and Meta-Analysis Protocols (INPLASY) on 15 November 2021 and was last updated on 15 November 2021 (registration number INPLASY2021110052).

Conflicts of interest:

None declared.

\section{INTRODUCTION}

Review question / Objective: Patient: patients with abdominal pain in emergency department; Intervention: speed bump sign;Comparison: acute appendicitis diagnosed by histology; $O$ : sensitivity, specificity, diagnostic odds ratio, likelihood ratio.

Condition being studied: Speed bumps are frequently used traffic device to slow down speed of vehicles and the "speed bump sign" is a clinical symptom with aggravated abdominal pain while driving over speed 
bumps, whose reason bases on irritation of inflamed peritoneum while moving. It is used by some doctors taking history from abdominal pain cases and the aggravation of pain while travelling over speed bumps is thought to be associated with an increased likelihood of acute appendicitis. However, the certainty of its evidence has not been validated.

\section{METHODS}

Participant or population: Patients with abdominal pain in emergency department.

Intervention: History taking about speed bump sign.

Comparator: Acute appendicitis diagnosed by histology.

Study designs to be included: Among the selected publications, pain triggered after pass the speed bumps as diagnosis of appendicitis, the true-positive, falsepositive, false-negative, true-negative data, adequate information for derivative parameters (sensitivity, specificity, negative predictive value, positive predictive value, and case numbers) should be contained in these studies.

Eligibility criteria: Non-relevant study, reports not retrieved, or unavailable full text

Information sources: We searched PubMed, Embase, Cochrane Library and Airiti Library independently for studies published before September 28, 2021. Keywords of "bump*" OR "speed bump" and "appendicitis" were used and no language limitation was applied. Besides, the relevant articles were also searched in some websites, such as xueshu.baidu.com, ResearchGate, Google Scholar, and Google.

Main outcome(s): Pooled sensitivity, specificity, diagnostic odds ratio, and positive/negative likelihood ratio, area under curve of hierarchical summary receiver operating characteristic curve.
Quality assessment / Risk of bias analysis: "Quality Assessment of Diagnostic Accuracy Studies 2" (QUADAS-2) was used to estimate quality of enrolled studies.

Strategy of data synthesis: We calculated the pooled estimates of sensitivity, specificity, positive and negative likelihood ratio with corresponding $95 \%$ confidence interval by the midas command in Stata 15 (StataCorp LLC., College Station, TX, USA). Cochran Q-statistic was used to assess statistical heterogeneity through 12 statistic and heterogeneity across studies was evaluated. The area under curve was used in describing overall accuracy as a potential summary of the hierarchical summary receiver operating characteristic curve. The diagnostic odds ratio was calculated by the metandi command in Stata 15. Fangan's Nomogram plot analysis for posttest probability was based on the pretest probability and likelihood ratio. Deeks' Funnel Plot asymmetry test was used for publication bias assessment and $p<0.05$ was considered statistically significant.

Subgroup analysis: Nil.

Sensitivity analysis: Nil.

Language: No restriction.

Country(ies) involved: Taiwan.

Keywords: Speed bump sign AND acute appendicitis.

Contributions of each author:

Author 1 - Ling Wang - Concept of the meta-analysis, database search, risk-ofbias appraisal, data extraction, interpretation of the data, and drafting of the manuscript.

Email: shellyjedi@gmail.com

Author 2 - Ching-Hsien Ling - Concept of the meta-analysis, database search, riskof-bias appraisal, data extraction, interpretation of the data, and drafting of the manuscript.

Email: Ics8931102@gmail.com 
Author 3 - Pei-Chun Lai - Statistical analyses, figure completion, and revision of the manuscript.

Email: debbie0613.lai@gmail.com

Author 4 - Yen-Ta Huang - Statistical analyses, figure completion, and revision of the manuscript.

Email: uncleda.huang@gmail.com 